\title{
Matched Queues with Flexible Customers and Impatient Customers
}

hengli liu ( $\nabla$ lhlhengli@163.com )

Yanshan University

\section{Research Article}

Keywords: Double-ended queue, matching queue, flexible customers, impatient customer, QBD process, RG-factorization

Posted Date: January 5th, 2022

DOI: https://doi.org/10.21203/rs.3.rs-1216110/v1

License: (c) (i) This work is licensed under a Creative Commons Attribution 4.0 International License. Read Full License 


\title{
Matched Queues with Flexible Customers and Impatient Customers
}

\author{
Heng-Li Liu \\ School of Economics and Management Sciences \\ Yanshan University, Qinhuangdao 066004, China \\ lhlhengli@163.com
}

December 31, 2021

\begin{abstract}
This paper studies a double-ended queue with four Poisson inputs and flexible customers, and its stability is guaranteed by customers' impatient behavior. We show that such a queue can be expressed as a quasi birth-and-death (QBD) process with infinitely many phases. For this purpose, we provide a detailed analysis for the QBD process, including the system stability, the stationary probability vector, the sojourn time, and so forth. Finally, numerical examples are employed to verify the correctness of our theoretical results, and demonstrate how the performance measures of this queue are influenced by key system parameters. We believe that the methodology and results described in this paper can be applied to analyze many practical issues, such as those encountered in sharing economy, organ transplantation, employee recruitment, online dating, and so on.
\end{abstract}

Keywords: Double-ended queue; matching queue; flexible customers; impatient customer; QBD process; RG-factorization.

\section{Introduction}

We consider a double-ended queueing system with flexible customers and impatient customers. There are two classes of customers, A-customers and B-customers. A-customers are divided into flexible customers and inflexible customers, denoted by Type 1 and Type 2. And B-customers are divided into flexible customers and inflexible customers, denoted 
by Class 2 and Class 1 . The A-customers of Type 1 and Type 2 enter the system, and queue up in their own order of arrival. When a B-customer of Class 1 arrives, she will match with an A-customers of Type 1. If the queue of A-customers of Type 1 is empty, the B-customer of Class 1 leaves. When a B-customer of Class 2 arrives, she will match with an A-customers of Type 1 or Type 2. The probability that a B-customer of Class 2 match with an A-customers of Type 1 is assumed to depend both on the number of A-customers of Type 1 and on the number of A-customers of Type 2. If both queues of A-customers of Type 1 and A-customers of Type 2 are empty, the B-customer of Class 2 leaves. For the A-customers and the B-customers, their match process follows a First-Come-First-Match discipline.

There are many different practical examples which can be characterized by doubleended queueing model with flexible customers. For example, Organ transplantation by Zenios [48], Boxma et al. [6], Stanford et al. [43], and Elalouf et al. [18]; taxi services by Giveen [20, 21], Kashyap [24, 25, 26], Bhat [5], Baik et al. [3], Shi and Lian [41], and Zhang et al. [49]; sharing economy by Cheng [16], Sutherland and Jarrahi [45], and Benjaafar and $\mathrm{Hu}$ [4]; transportation by Browne et al. [9] and Baik et al. [3]; assembly systems by Hopp and Simon [22], Som et al. [42], and Ramachandran and Delen [39]; inventory management by Sasieni [40], Porteus [38], and Axsäter [2]; health care by Pandey and Gangeshwer [34]; multimedia synchronization by Steinmetz [44] and Parthasarathy et al. [35]; housing allocations systems by Talreja and Whitt [46] and Johnson [23]; online dating by Chen et al. [15], Pizzato et al. [37] and Epstein and Robert [19], and so on.

In recent years, academic research related to double-ended queue has grown rapidly. In these queueing system, matching process is aimed at two classes of users: customers and servers. Kashyap [25] considers the system in which there is limited waiting space both for customers and for taxis, and that the taxis 'serve' the customers in bulk. Conolly et al. [17] studied the effect of impatience behavior of customers and taxis primarily in the context of double-ended queues under the common setting of Poisson arrivals and exponential impatience times. Liu et al. [31] considered some diffusion models for double-ended queues where the sellers and buyers arrive according to independent renewal processes. Liu et al. [29] studied the double-ended queue under the assumption that A-customers and B-customers arrive with Markovian arrival processes (MAPs) and exponential impatience times. Liu et al. [30] analyzed a more general matched queue with matching batch pair $(m, n)$ and impatient customers, where the arrivals of A- and B-customers are both 
Poisson processes. Büke and Chen $[10,11]$ studied stabilizing policies for probabilistic matching systems and used fluid and diffusion approximations to analyze the system which considering impatient behavior.

What's more, according to different properties, these two classes are further divided into several different types. Caldentey et al. [13] considered a bipartite matching of multitype customers to multi-type servers. Adan and Weiss [1] described multi-type customers to multi-type servers by a multi-dimensional countable Markov chain, obtained ergodicity condition, and derived its stationary distribution of product-form for matching rate. Bušić et al. [12] studied the stability of the associated discrete time Markov chain under various admissible matching policies for systems where customers and servers form a bipartite graph. Gurvich and Ward [47] considered optimal control of the matching of different classes of items that arrive randomly over time. Mairesse and Moyal [32] generalized the bipartite matching model and developed necessary conditions for matching networks with general topology. Perlman et al. [36] provided and analyzed a general model of the flexible-resource queueing system, and then focused on its application in the context of live kidney transplantation. Castro et al. [14] studied a parallel matching queue with reneging, and derived explicit product forms of the steady state distributions of this system.

In this paper, we studied a parallel matching queue where A-customers and B-customers arrive randomly over time. A-customers queue up in the system, and may abandon the system as time goes by. But B-customers arrival have zero patience and leave the system if they cannot be matched immediately. This queueing system is expressed as a leveldependent QBD process with infinitely many phases in which its analysis is extremelly challenging. We via RG-factorization to obtain stationary probability distribution which can be the basis of stationary queue length and sojourn time. Readers may refer to Li [27], Li and Cao [28] about the RG-factorizations.

We summarize the main contributions of this paper as follows:

(1) We describe a more general matched queue with with flexible customers and impatient customers, and also the customers' impatient behavior is to guatantee the stability of the system.

(2) We express the matched queue with flexible customers as a level-dependent QBD process with infinitely many phases, and apply the RG-factorizations by $\mathrm{Li}[27]$ to obtain the stationary probability vector, which is used for analyzing the average 
stationary queue lengths of the A-customers of Type 1 and Type 2.

(3) We study the average sojourn time of any A-customer of Type 1 and Type 2 by using a phase-type distribution, whose computation is established in terms of the RG-factorizations given in $\mathrm{Li}[27]$.

(4) We use some numerical examples to verify the correctness of our theoretical results, and indicate how the performance measures of the double-ended queue with flexible customers are influenced by key system parameters. In addition, the numerical results are also given a simple but interesting discussion by means of the coupling method of Markov processes.

The structure of this paper is organized as follows. Section 2 describes a double-ended queue with flexible customers, four Poisson inputs and customers' impatient behaviors. Section 3 shows that the double-ended queue can be expressed as a new QBD process with infinitely many phases. By using the QBD process with infinitely many phases, we obtain some stable conditions for the double-ended queues with flexible customers. Section 4 studies the stationary probability vector of the QBD process, so that we can compute the average stationary queue lengths of the A-customers of Type 1 and Type 2. Section 5 discusses the sojourn time of each arriving A-customer of Type 1 and Type 2 respectively, and provides a computational method of $\mathrm{PH}$ distribution of infinite sizes to deal with the average sojourn time. Section 6 uses some numerical examples to indicate how the performance measures of the double-ended queue are influenced by key system parameters. Finally, Section 7 gives some concluding remarks.

\section{Model Description}

In this section, we describe a more general double-ended queue with flexible customers, four Poisson inputs and customers' impatient behaviors, and also introduce operational mechanism, system parameters and basic notation.

In the proposed queue, there are two kinds of customers, called A-customers and B-customers. A-customers are divided into flexible customers and inflexible customers, denoted by Type 1 and Type 2. And B-customers are divided into flexible customers and inflexible customers, denoted by Class 2 and Class 1. If an arriving A-customer and an 
arriving B-customer match, they will constitute a group and leave the queueing system immediately. Figure 1 provides a physical illustration for such a double-ended queue with flexible customers.

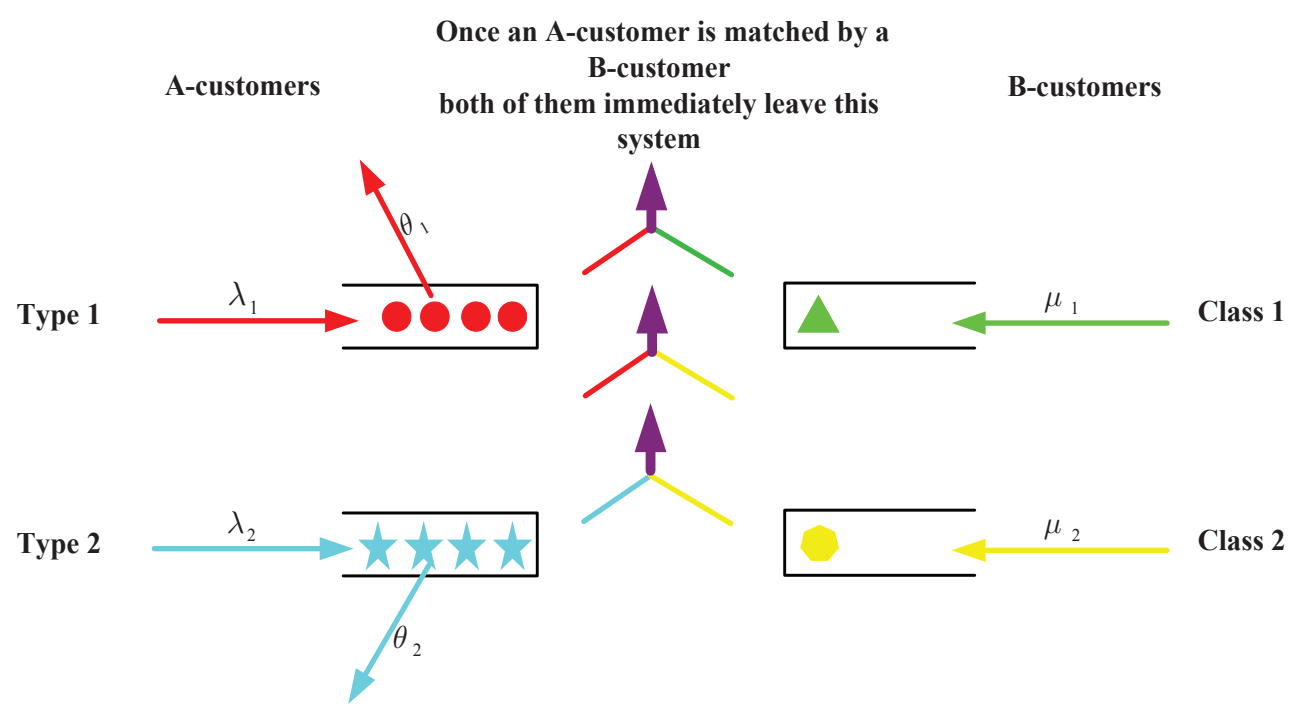

Figure 1: A physical illustration of the matching queue

Now, we provide a more detailed description for the double-ended queue with flexible customers as follows:

(1) Arrival processes. The A-customers of Type 1 and Type 2 arrive at the queueing system according to a Poisson process with rate $\lambda_{1}$ and $\lambda_{2}$, respectively. Similarly, the B-customers of Class 1 and Class 2 arrive at the queueing system according to a Poisson process with rate $\mu_{1}$ and $\mu_{2}$, respectively.

(2) Matched processes. The A-customers of Type 1 and Type 2 enter the system, and queue up in their own order of arrival. When a B-customer of Class 1 arrives, she will match with an A-customers of Type 1. If the queue of A-customers of Type 1 is empty, the B-customer of Class 1 leaves. When a B-customer of Class 2 arrives, she will match with an A-customers of Type 1 or Type 2 . The probability that a B-customer of Class 2 match with an A-customers of Type 1 is assumed to depend both on the number of A-customers of Type 1 and on the number of A-customers of Type 2. If both queues of A-customers of Type 1 and A-customers of Type 2 are empty, the B-customer of Class 2 leaves. For the A-customers and the B-customers, 
their match process follows a First-Come-First-Match discipline. We assume the two waiting spaces of A-customers of Type 1 and Type 2 are both infinite.

(3) Impatient behavior. If an A-customer of Type 1 (resp. an A-customer of Type 2 ) stays in the queueing system for a long time, then she will show some impatience. To capture this phenomenon, we assume that the impatient time, which is defined as the longest time that a customer stays in the buffer before leaving, of an A-customer of Type 1 (resp. an A-customer of Type 2) is exponentially distributed with rate $\theta_{1}$ (resp. $\theta_{2}$ ), which is hereafter called impatient rate, $\theta_{1}, \theta_{2}>0$.

We assume that all the random variables defined above are independent of each other.

Remark 1. (a) The matching discipline given in Assumption (2) indicates that the Bcustomers are zero impatience.

(b) The customers' impatient behavior given in Assumption (3) is used to ensure stability of the matched queue.

\section{A QBD Process with Infinitely Many Phases}

In this section, we show that the double-ended queue with flexible customers can be expressed as a new QBD process with infinitely many phases. By using the QBD process, we obtain some stable conditions of the double-ended queue with flexible customers.

We denote by $N_{1}(t)$ and $N_{2}(t)$ the numbers of the A-customers of Type 1 and Type 2 in the double-ended queue at time $t \geq 0$, respectively. Let $d_{i j}$ be the probability that an arriving B-customer of Class 2 match with an A-customer of Type 1 when the system is in state $\left(N_{1}(t)=i, N_{2}(t)=j\right)$. We assume that $d_{0 j}=0$ for all $j \geq 1$, since when $N_{1}(t)=0$, an arriving B-customer of Class 2 only matches with an A-customer of Type 2. And we assume that $d_{i 0}=1$ for all $i \geq 1$, since when $N_{2}(t)=0$, an arriving B-customer of Class 2 matches with probability 1 with an A-customer of Type 1. Moreover, we assume that $d_{i j}=i /(i+j)$ for $i \geq 1$ and $j \geq 1$.

The double-ended queue with flexible customers can be modeled as a two-dimensional Markov process $\left\{\left(N_{1}(t), N_{2}(t)\right), t \geq 0\right\}$. State space of the Markov process $\left\{\left(N_{1}(t), N_{2}(t)\right), t \geq 0\right\}$ is given by

$$
\Omega=\{0,1,2, \ldots\} \times\{0,1,2, \ldots\} .
$$


The states of this space are arranged in the following order:

$$
\left\{S_{0}, S_{1}, S_{2}, \ldots\right\}, S_{j}=\{(0, j),(1, j),(2, j),(3, j), \ldots\} \text {. }
$$

For convenience, the state transition relations of the Markov process $\left\{\left(N_{1}(t), N_{2}(t)\right), t \geq 0\right\}$ is depicted in Figure 2. It is easy to see from Figure 2 that the Markov process $\left\{\left(N_{1}(t), N_{2}(t)\right)\right.$, $t \geq 0\}$ is a new QBD process with infinitely many phases.

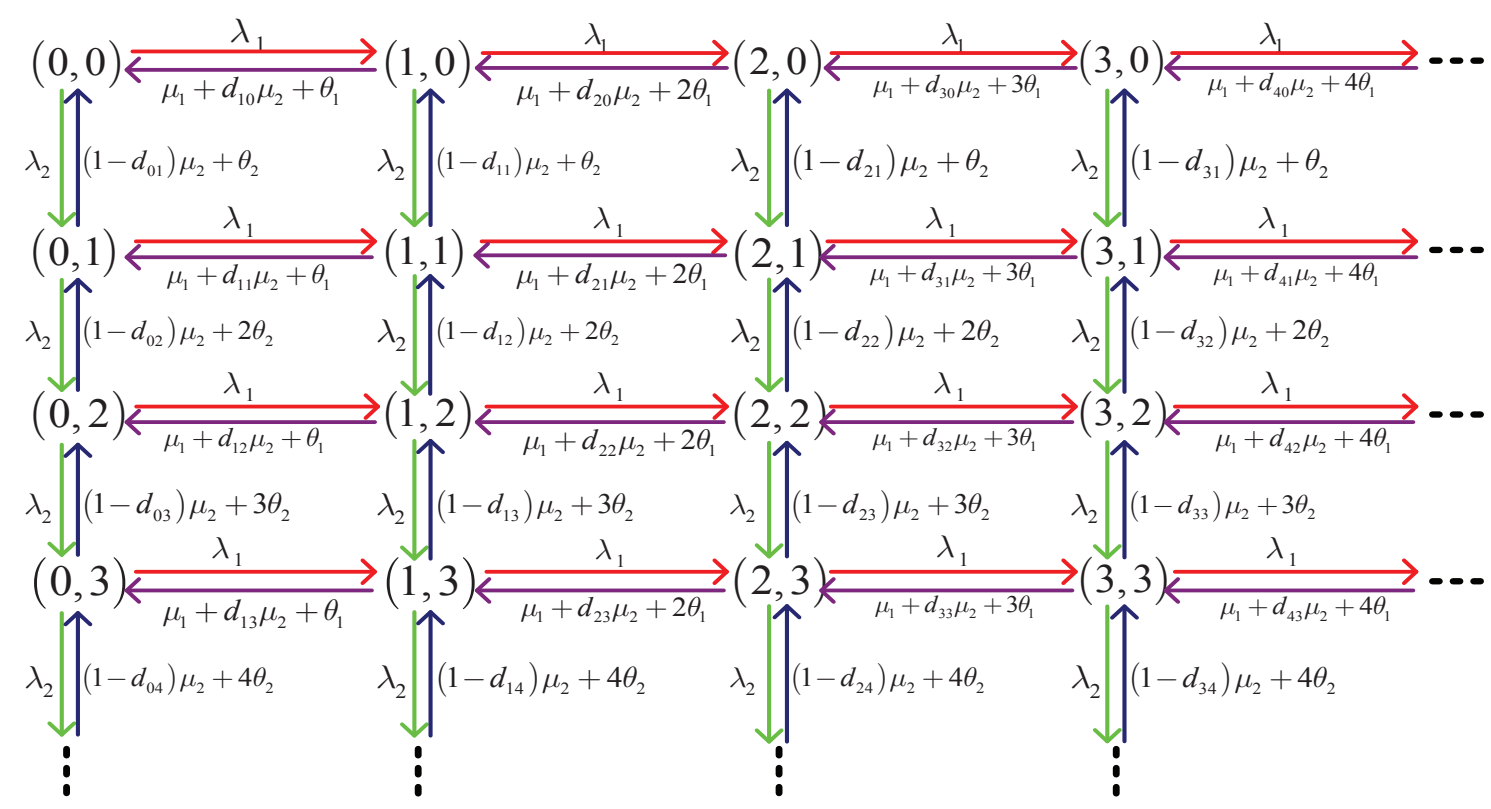

Figure 2: The state transition relations of the QBD process

Based on Figure 2, the infinitesimal generator of the QBD process $\left\{\left(N_{1}(t), N_{2}(t)\right)\right.$, $t \geq 0\}$ is given by

$$
Q=\left(\begin{array}{cccccc}
A_{1}^{(0)} & A_{0}^{(0)} & & & & \\
A_{2}^{(1)} & A_{1}^{(1)} & A_{0}^{(1)} & & & \\
& A_{2}^{(2)} & A_{1}^{(2)} & A_{0}^{(2)} & & \\
& & A_{2}^{(3)} & A_{1}^{(3)} & A_{0}^{(3)} & \\
& & & \ddots & \ddots & \ddots
\end{array}\right)
$$

where

$$
A_{0}^{(j)}=\left(\begin{array}{cccc}
\lambda_{2} & & & \\
& \lambda_{2} & & \\
& & \lambda_{2} & \\
& & & \ddots
\end{array}\right), j \geq 0,
$$




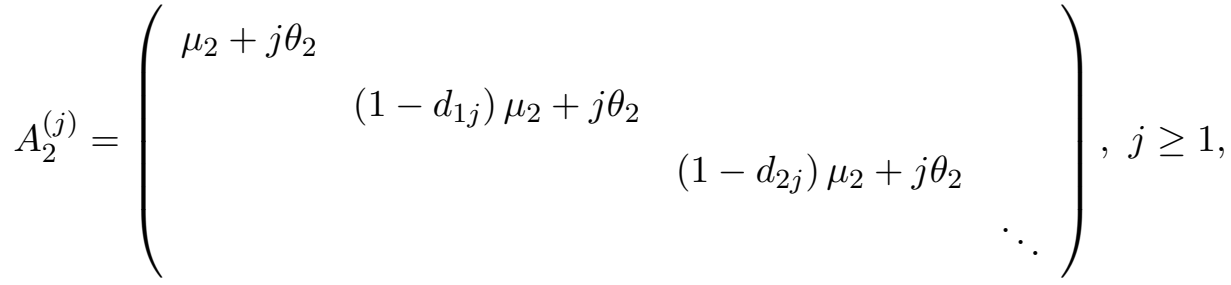

$$
\begin{aligned}
& A_{1}^{(j)}=\left(\begin{array}{ccccc}
A_{1,1} & \lambda_{1} & & \\
\mu_{1}+d_{1 j} \mu_{2}+\theta_{1} & A_{1,2} & \lambda_{1} & \\
& \mu_{1}+d_{2 j} \mu_{2}+2 \theta_{1} & A_{1,3} & \lambda_{1} & \\
& & \ddots & \ddots & \ddots
\end{array}\right), j \geq 0, \\
& A_{1,1}=-\left(\lambda_{1}+\lambda_{2}+\left(1-d_{0 j}\right) \mu_{2}+j \theta_{2}\right), d_{0 j}=\left\{\begin{array}{c}
1, j=0 \\
0, j \geq 1
\end{array},\right. \\
& A_{1, i}=-\left(\lambda_{1}+\lambda_{2}+\mu_{1}+\mu_{2}+(i-1) \theta_{1}+j \theta_{2}\right), i \geq 2 .
\end{aligned}
$$

The following theorem provides a necessary and sufficient condition for the stability of the double-ended queue with $\left(\theta_{1}, \theta_{2}\right)>0$.

Theorem 1. If $\left(\theta_{1}, \theta_{2}\right)>0$, then the $Q B D$ process $Q$ must be irreducible and positive recurrent. Thus the double-ended queue is stable.

Proof. It is easy to see the irreducibility of the QBD process $Q$ through observing Figure 2 , and using the four Poisson inputs, matching probability $d_{i j}$ and the two exponential impatient times with $\left(\theta_{1}, \theta_{2}\right)>0$.

At the same time, it is not difficult to find that the QBD process $Q$ is bidirectional infinite in both level and phase. Thus, we need to prove the QBD process $Q$ in both level and phase are positive recurrent.

In order to prove the QBD process $Q$ in overall level is positive recurrent, it is key to find some necessary and sufficient condition for the stability of the QBD process $Q$ by means of the mean drift technique by Neuts [33].

For the QBD process $Q$, let $A_{j}=A_{0}^{(j)}+A_{1}^{(j)}+A_{2}^{(j)}$ in level $j$. Then for $j \geq 1$, we obtain

$$
A_{j}=\left(\begin{array}{ccccc}
a_{0,0} & a_{0,1} & & & \\
a_{1,0} & a_{1,1} & a_{1,2} & & \\
& a_{2,1} & a_{2,2} & a_{2,3} & \\
& & \ddots & \ddots & \ddots
\end{array}\right),
$$


where

$$
\begin{gathered}
a_{i, i+1}=\lambda_{1}, i \geq 0, \\
a_{i, i-1}=\mu_{1}+d_{i j} \mu_{2}+i \theta_{1}, i \geq 1, \\
a_{i, i}=-\left(\lambda_{1}+\mu_{1}+d_{i j} \mu_{2}+i \theta_{1}\right), i \geq 1, a_{0,0}=-\lambda_{1} .
\end{gathered}
$$

Let $\beta=\left(\beta_{1}, \beta_{2}, \beta_{3}, \ldots\right)$ be the stationary probability vector of the Markov process $A_{j}$. Thus,

$$
\beta A_{j}=\mathbf{0}, \beta \mathbf{e}=1 .
$$

Note that $\beta>0$, since the Markov process $A_{j}$ is irreducible.

Once the stationary probability vector $\beta$ is obtained, we can compute the (upward and downward) mean drift rates of the QBD process $Q$. From level $j$ to level $j+1$, the upward mean drift rate is given by

$$
\beta A_{0}^{(j)} \mathbf{e}=\lambda_{2}\left(\beta_{1}+\beta_{2}+\beta_{3}+\cdots\right)=\lambda_{2} .
$$

Similarly, from level $j$ to level $j-1$, the downward mean drift rate is given by

$$
\beta A_{2}^{(j)} \mathbf{e}=\mu_{2}+j \theta_{2}-\sum_{i=2}^{+\infty} \beta_{i} d_{(i-1) j} \mu_{2} .
$$

Note that $j$ is a positive integer, $\lambda_{2}>0, \mu_{2}>0$ and $\theta_{2}>0$, it is easy to check that if $j>\left(\lambda_{2}-\mu_{2}\right) / \theta_{2}$, then $\beta A_{0}^{(j)} \mathbf{e}<\beta A_{2}^{(j)} \mathbf{e}$. Therefore, the QBD process $Q$ in overall level is positive recurrent due to the fact that the mean drift rates: $\beta A_{0}^{(j)} \mathbf{e}<\beta A_{2}^{(j)} \mathbf{e}$ for a bigger positive integer $j$, this can hold because $j$ goes to infinity. Then $\beta=\left(\beta_{1}, \beta_{2}, \beta_{3}, \ldots\right)$ is the stationary probability vector of the Markov process $A_{j}$.

Moreover, in order to prove the QBD process $Q$ in phase at a certain level (that is, $A_{j}$ with the fixed level $j$ ) is positive recurrent, it is key to find some necessary and sufficient condition for the stability of the QBD process $A_{j}$ by means of Neuts' method (i.e., the mean drift technique).

Now, we can compute the (upward and downward) mean drift rates of the QBD process $A_{j}$. For $i \geq 1$, from phase $i$ to phase $i+1$, the upward mean drift rate is given by

$$
a_{i, i+1}=\lambda_{1}
$$

Similarly, from phase $i$ to $i-1$, the downward mean drift rate is given by

$$
a_{i, i-1}=\mu_{1}+d_{i j} \mu_{2}+i \theta_{1} \text {. }
$$


Note that $i$ is a positive integer, $\lambda_{1}>0, \mu_{1}>0, \mu_{2}>0$ and $\theta_{2}>0$, it is easy to check that if $i>\left(\lambda_{1}-\mu_{1}-\mu_{2}\right) / \theta_{2}$, then $a_{i, i-1}>a_{i, i+1}$. Therefore, the QBD process $Q$ in phase is positive recurrent due to the fact that $a_{i, i-1}>a_{i, i+1}$ for a bigger positive integer $i$, this can hold because $i$ goes to infinity.

Combining with above proof of positive recurrent for QBD process $Q$ in both level and phase, the double-ended queue is stable. This completes the proof.

\section{The Stationary Queue Length}

In this section, we first provide a matrix-product expression for the stationary probability vector of the $\mathrm{QBD}$ process by means of the RG-factorization. Then we compute two average stationary queue lengths for the A-customers of Type 1 and Type 2, respectively.

We write

$$
p_{i, j}(t)=P\left\{N_{1}(t)=i, N_{2}(t)=j\right\} .
$$

Since the level-dependent QBD process is stable, we have

$$
\pi_{i, j}=\lim _{t \rightarrow+\infty} p_{i, j}(t)
$$

For $k \geq 0$, we write

$$
\pi_{k}=\left(\pi_{0, k}, \pi_{1, k}, \pi_{2, k}, \ldots\right),
$$

and

$$
\pi=\left(\pi_{0}, \pi_{1}, \pi_{2}, \ldots\right)
$$

Note that the $\mathrm{QBD}$ process $Q$ is level-dependent, thus we need to apply the RGfactorization given in $\mathrm{Li}[27]$ to calculate their stationary probability vectors. To this end, we need to introduce the $U$-, $R$ - and $G$-measures for the QBD process $Q$.

For the QBD process $Q$, we define the $U L$-type $U$-, $R$ - and $G$-measures as

$$
\begin{gathered}
U_{k}=A_{1}^{(k)}+A_{0}^{(k)}\left(-U_{k+1}^{-1}\right) A_{2}^{(k+1)}, \quad k \geq 0, \\
R_{k}=A_{0}^{(k)}\left(-U_{k+1}^{-1}\right), \quad k \geq 0,
\end{gathered}
$$

and

$$
G_{k}=\left(-U_{k}^{-1}\right) A_{2}^{(k)}, \quad k \geq 1 .
$$

On the other hand, it is well-known that the matrix sequence $\left\{R_{k}, k \geq 0\right\}$ is the minimal nonnegative solution to the system of nonlinear matrix equations 


$$
A_{0}^{(k)}+R_{k} A_{1}^{(k+1)}+R_{k} R_{k+1} A_{2}^{(k+2)}=0, \quad k \geq 0,
$$

and the matrix sequence $\left\{G_{k}, k \geq 1\right\}$ is the minimal nonnegative solution to the system of nonlinear matrix equations

$$
A_{0}^{(k)} G_{k+1} G_{k}+A_{1}^{(k)} G_{k}+A_{2}^{(k)}=0, \quad k \geq 1 .
$$

Once the matrix sequence $\left\{R_{k}, k \geq 0\right\}$ or $\left\{G_{k}, k \geq 1\right\}$ is given, for $k \geq 0$

$$
\begin{aligned}
U_{k} & =A_{1}^{(k)}+A_{0}^{(k)}\left(-U_{k+1}^{-1}\right) A_{2}^{(k+1)} \\
& =A_{1}^{(k)}+R_{k} A_{2}^{(k+1)} \\
& =A_{1}^{(k)}+A_{0}^{(k)} G_{k+1} .
\end{aligned}
$$

For the QBD process $Q$, by following Chapters 1 and 2 of $\mathrm{Li}$ [27] or Li and Cao [28], the $U L$-type RG-factorization is given by

$$
Q=\left(I-R_{U}\right) U_{D}\left(I-G_{L}\right)
$$

where

$$
R_{U}=\left(\begin{array}{ccccc}
0 & R_{0} & & & \\
& 0 & R_{1} & & \\
& & 0 & R_{2} & \\
& & & 0 & \ddots \\
& & & \ddots
\end{array}\right), \quad G_{L}=\left(\begin{array}{ccccc}
0 & & & & \\
G_{1} & 0 & & & \\
& G_{2} & 0 & & \\
& & G_{3} & 0 & \\
& & & \ddots & \ddots
\end{array}\right) .
$$

The following theorem expresses the stationary probability vector $\pi=\left(\pi_{0}, \pi_{1}, \pi_{2}, \ldots\right)$ of the QBD process $Q$.

Theorem 2. The stationary probability vector $\pi$ of the level-dependent $Q B D$ process $Q$ is given by

$$
\pi_{0}=c \widetilde{\pi}_{0}
$$

and

$$
\pi_{k}=c \widetilde{\pi}_{0} R_{0} R_{1} \cdots R_{k-1}, k \geq 1,
$$

where the boundary vector $\widetilde{\pi}_{0}$ is uniquely determined by the system of linear equation

$$
\widetilde{\pi}_{0} A_{1}^{(0)}+\widetilde{\pi}_{0} R_{0} A_{2}^{(1)}=0
$$


and the positive constant $c$ is uniquely given by

$$
c=\frac{1}{\widetilde{\pi}_{0} \mathbf{e}+\sum_{k=1}^{\infty} \widetilde{\pi}_{0} R_{0} R_{1} \cdots R_{k-1} \mathbf{e}} .
$$

Proof. The proof is easy through checking that $\pi$ satisfies the system of linear equations $\pi Q=\mathbf{0}$ and $\pi \mathbf{e}=1$. To this end, we consider the following two different cases:

Case one: $k=0$. In this case, we need to check that

$$
\pi_{0} A_{1}^{(0)}+\pi_{1} A_{2}^{(1)}=0,
$$

by means of $\pi_{0}=c \widetilde{\pi}_{0}, \pi_{1}=c \widetilde{\pi}_{0} R_{0}$.

Case two: $k \geq 1$. In this case, we need to check that

$$
\pi_{k} A_{0}^{(k)}+\pi_{k+1} A_{1}^{(k+1)}+\pi_{k+2} A_{2}^{(k+2)}=0 .
$$

Since $\pi_{k}=c \widetilde{\pi}_{0} R_{0} R_{1} \cdots R_{k-1}, \pi_{k+1}=c \widetilde{\pi}_{0} R_{0} R_{1} \cdots R_{k}$ and $\pi_{k+2}=c \widetilde{\pi}_{0} R_{0} R_{1} \cdots R_{k+1}$, we obtain

$$
\begin{aligned}
& \pi_{k} A_{0}^{(k)}+\pi_{k+1} A_{1}^{(k+1)}+\pi_{k+2} A_{2}^{(k+2)} \\
= & c \widetilde{\pi}_{0} R_{0} R_{1} \cdots R_{k-1} A_{0}^{(k)}+c \widetilde{\pi}_{0} R_{0} R_{1} \cdots R_{k} A_{1}^{(k+1)}+c \widetilde{\pi}_{0} R_{0} R_{1} \cdots R_{k+1} A_{2}^{(k+2)} \\
= & c \widetilde{\pi}_{0} R_{0} R_{1} \cdots R_{k-1}\left(A_{0}^{(k)}+R_{k} A_{1}^{(k+1)}+R_{k} R_{k+1} A_{2}^{(k+2)}\right)=0
\end{aligned}
$$

by means of (3).

Let $\mathbf{N}$ be the set of all nature number, i.e., $\mathbf{N}=\{0,1,2, \ldots\}$. Note that $\pi \mathbf{e}=1$, we compute

$$
\sum_{k \in \mathbf{N}} \pi_{k} \mathbf{e}=1
$$

by means of $\pi_{0}=c \widetilde{\pi}_{0}, \pi_{k}=c \widetilde{\pi}_{0} R_{0} R_{1} \cdots R_{k-1}$ for $k \geq 1$. Thus we have

$$
\begin{aligned}
1 & =\sum_{k \in \mathbf{N}} \pi_{k} \mathbf{e}=\pi_{0} \mathbf{e}+\sum_{k=1}^{\infty} \pi_{k} \mathbf{e} \\
& =c \widetilde{\pi}_{0} \mathbf{e}+\sum_{k=1}^{\infty} c \widetilde{\pi}_{0} R_{0} R_{1} \cdots R_{k-1} \mathbf{e} \\
& =c\left(\widetilde{\pi}_{0} \mathbf{e}+\sum_{k=1}^{\infty} \widetilde{\pi}_{0} R_{0} R_{1} \cdots R_{k-1} \mathbf{e}\right),
\end{aligned}
$$

which gives the positive constant $c$ in (9). This completes the proof.

In the remainder of this section, we provide average stationary queue lengths of the double-ended queue by means of the stationary probability vector of the QBD process. 
Note that the double-ended queue must be stable for $\left(\theta_{1}, \theta_{2}\right)>0$. Thus, we denote by $\mathcal{Q}^{(1)}$ and $\mathcal{Q}^{(2)}$ the stationary queue lengths of the A-customers of Type 1 and Type 2 , respectively.

By using Theorem 2, we can obtain the average stationary queue length of the Acustomers of Type 1 is

$$
E\left[\mathcal{Q}^{(1)}\right]=\sum_{i=1}^{+\infty} \sum_{j=0}^{+\infty} i \pi_{i, j},
$$

and the average stationary queue length of the A-customers of Type 2 is

$$
E\left[\mathcal{Q}^{(2)}\right]=\sum_{j=1}^{+\infty} \sum_{i=0}^{+\infty} j \pi_{i, j}
$$

\section{The Sojourn Time}

In this section, we analyze two expected sojourn time of each arriving A-customer of Type 1 and Type 2 in the double-ended queue, respectively.

\subsection{Sojourn Time of the A-customers of Type 2}

When the double-ended queue is stable, we denote by $W^{(2)}$ the sojourn time of any arriving A-customer of Type 2 .

For a new arrival, his sojourn time can be regarded as the first passage time that the QBD process $\left\{\left(N_{1}(t), N_{2}(t)\right), t \geq 0\right\}$ with absorbing states $\{(0,0),(1,0),(2,0), \ldots\}$ and on a state sub-space

$$
\{\{0,1,2, \ldots\} \times\{1,2,3, \ldots\}\}
$$

begins from state $(i, j)$ with probability $\pi_{i, j}$ for $i \geq 0, j \geq 1$ until it finally enters the absorption state $\{(0,0),(1,0),(2,0), \ldots\}$. Note that the new QBD process with the absorption state $\{(0,0),(1,0),(2,0), \ldots\}$ is depicted in Figure 3 , thus the infinitesimal generator of the new QBD process with the absorption state $\{(0,0),(1,0),(2,0), \ldots\}$ is given by 


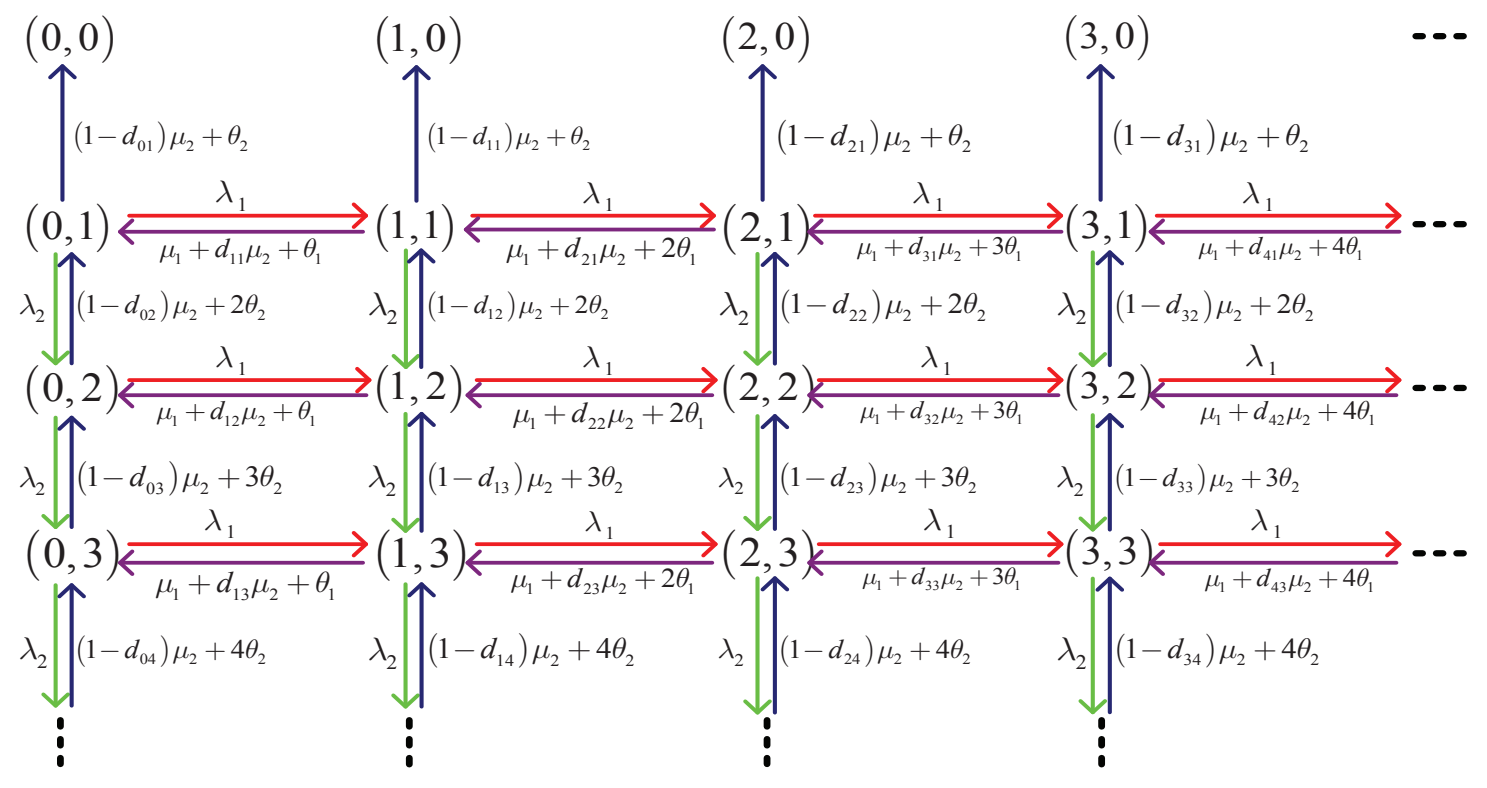

Figure 3: The state transition relations of the QBD process

$$
\Theta=\left(\begin{array}{cccccc}
0 & \mathbf{0} & \mathbf{0} & \mathbf{0} & \mathbf{0} & \cdots \\
A_{2}^{(1)} \mathbf{e} & A_{1}^{(1)} & A_{0}^{(1)} & & & \\
& A_{2}^{(2)} & A_{1}^{(2)} & A_{0}^{(2)} & & \\
& & A_{2}^{(3)} & A_{1}^{(3)} & A_{0}^{(3)} & \\
& & & \ddots & \ddots & \ddots
\end{array}\right)
$$

where

$$
\begin{aligned}
& A_{0}^{(j)}=\left(\begin{array}{cccc}
\lambda_{2} & & & \\
& \lambda_{2} & & \\
& & \lambda_{2} & \\
& & & \ddots
\end{array}\right), j \geq 1
\end{aligned}
$$

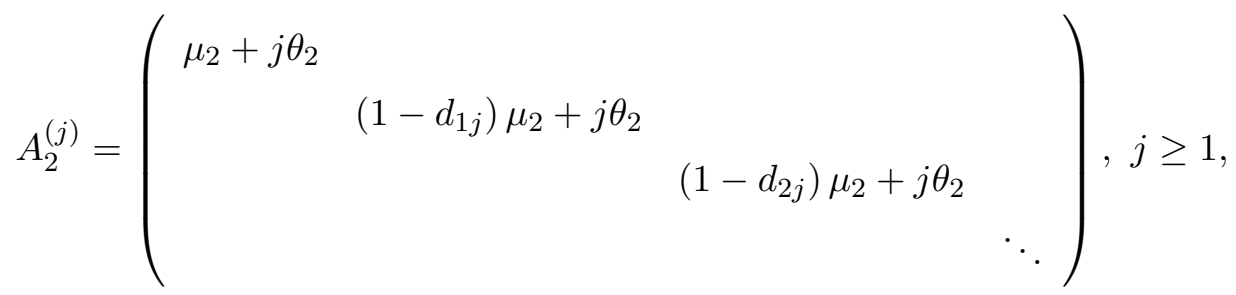




$$
\begin{aligned}
& A_{1}^{(j)}=\left(\begin{array}{ccccc}
A_{1,1} & \lambda_{1} & & \\
\mu_{1}+d_{1 j} \mu_{2}+\theta_{1} & A_{1,2} & \lambda_{1} & & \\
& \mu_{1}+d_{2 j} \mu_{2}+2 \theta_{1} & A_{1,3} & \lambda_{1} & \\
& & \ddots & \ddots & \ddots
\end{array}\right), j \geq 1, \\
& A_{1,1}=-\left(\lambda_{1}+\lambda_{2}+\mu_{2}+j \theta_{2}\right), \\
& A_{1, i}=-\left(\lambda_{1}+\lambda_{2}+\mu_{1}+\mu_{2}+(i-1) \theta_{1}+j \theta_{2}\right), i \geq 2 \text {. }
\end{aligned}
$$

Let

$$
T=\left(\begin{array}{cccccc}
A_{1}^{(1)} & A_{0}^{(1)} & & & & \\
A_{2}^{(2)} & A_{1}^{(2)} & A_{0}^{(2)} & & & \\
& A_{2}^{(3)} & A_{1}^{(3)} & A_{0}^{(3)} & & \\
& & A_{2}^{(4)} & A_{1}^{(4)} & A_{0}^{(4)} & \\
& & & \ddots & \ddots & \ddots
\end{array}\right), \quad T^{0}=\left(\begin{array}{c}
A_{2}^{(1)} \mathbf{e} \\
0 \\
0 \\
0 \\
\vdots
\end{array}\right) .
$$

Then (12) can be rewritten as

$$
\Theta=\left(\begin{array}{cc}
0 & 0 \\
T^{0} & T
\end{array}\right)
$$

Here, $T \mathbf{e}+T^{0}=0$.

Let $\left(\alpha_{0}, \vec{\alpha}\right)$ denote the initial probability distribution of the QBD process $\Theta$ with the absorption state $\{(0,0),(1,0),(2,0), \ldots\}$, where $\vec{\alpha}=\left(\pi_{1}, \pi_{2}, \ldots\right)$ and $\alpha_{0}=1-\vec{\alpha} \mathbf{e}=\pi_{0}$.

The following theorem uses the phase-type distribution of infinite size to provide expression for the probability distribution of the sojourn time $W^{(2)}$.

Theorem 3. If the initial probability distribution of the $Q B D$ process $\Theta$ with the absorption state $\{(0,0),(1,0),(2,0), \ldots\}$ is $\left(\alpha_{0}, \vec{\alpha}\right)$, then the probability distribution of the sojourn time $W^{(2)}$ is phase-type with an irreducible representation $(\vec{\alpha}, T)$, that is,

$$
F_{W^{(2)}}(t)=P\left(W^{(2)} \leq t\right)=1-\vec{\alpha} \exp (T t) \mathbf{e}, \quad t \geq 0 .
$$

Proof. For $i=0,1,2, \ldots, j=1,2,3, \ldots$, we write

$$
q_{i, j}(t)=P\left\{N_{1}(t)=i, N_{2}(t)=j\right\},
$$

for $j=1,2,3, \ldots$, we write

$$
q_{j}(t)=\left(q_{0, j}(t), q_{1, j}(t), q_{2, j}(t), \ldots\right)
$$


and

$$
q(t)=\left(q_{1}(t), q_{2}(t), q_{3}(t), \ldots\right)
$$

For the probability vector $q(t)$, it follows from the Chapman-Kolmogorov forward equation that

$$
\frac{d}{d t} q(t)=q(t) T
$$

with the initial condition

$$
q(0)=\vec{\alpha}
$$

It follows from (14) and (15) that

$$
q(t)=\vec{\alpha} e^{T t}
$$

Thus, we obtain

$$
F_{W^{(2)}}(t)=P\left(W^{(2)} \leq t\right)=1-q(t) \mathbf{e}=1-\vec{\alpha} \exp (T t) \mathbf{e}, t \geq 0
$$

This completes the proof.

In the remainder of this section, we compute the mean $E\left[W^{(2)}\right]$ of the sojourn time $W^{(2)}$ of each arriving A-customer of Type 2 by means of the Laplace-Stieltjes transform and the RG-factorization.

We denote by $f(s)$ the Laplace-Stieltjes transform of the distribution function $F_{W^{(2)}}(t)$ or the random variable $W^{(2)}$. Thus, we have

$$
\begin{aligned}
f(s) & =\int_{0}^{\infty} e^{-s t} d F_{W^{(2)}}(t) \\
& =1-\vec{\alpha} \mathbf{e}+\vec{\alpha}(s I-T)_{\min }^{-1} T^{0},
\end{aligned}
$$

where $I$ denotes an identity matrix of infinite size, and $(s I-T)_{\min }^{-1}$ is the minimal nonnegative inverse of the matrix $s I-T$ of infinite size. See Li and Cao [28] and Li [27] for more details. It is easy to see that

$$
E\left[W^{(2)}\right]=-\frac{d}{d s} f(s)_{\mid s=0}=\vec{\alpha}\left[(s I-T)_{\min }^{-2}\right]_{\mid s=0} T^{0}=\vec{\alpha}(-T)_{\min }^{-1} \mathbf{e}
$$

by using $T \mathbf{e}+T^{0}=0$ and $(-T)_{\min }^{-1}(-T) \mathbf{e}=\mathbf{e}$.

In computation of the mean $E\left[W^{(2)}\right]$, it is easy to see that the key is how to deal with the minimal nonnegative inverse $(-T)_{\min }^{-1}$ of the matrix $-T$ of infinite size. To this end, 
we develop an effective procedure to compute the minimal nonnegative inverse $(-T)_{\min }^{-1}$ by means of the RG-factorization.

For the QBD process $T$, we define the $U L$-type $\mathbf{U}$-, $\mathbf{R}$ - and $\mathbf{G}$-measures as

$$
\begin{gathered}
\mathbf{U}_{j}=A_{1}^{(j)}+A_{0}^{(j)}\left(-\mathbf{U}_{j+1}^{-1}\right) A_{2}^{(j+1)}, j \geq 1, \\
\mathbf{R}_{j}=A_{0}^{(j)}\left(-\mathbf{U}_{j+1}^{-1}\right), j \geq 1,
\end{gathered}
$$

and

$$
\mathbf{G}_{j}=\left(-\mathbf{U}_{j}^{-1}\right) A_{2}^{(j)}, j \geq 2 .
$$

On the other hand, note that the matrix sequence $\left\{\mathbf{R}_{j}, j \geq 1\right\}$ is the minimal nonnegative solution to the system of nonlinear matrix equations

$$
A_{0}^{(j)}+\mathbf{R}_{j} A_{1}^{(j+1)}+\mathbf{R}_{j} \mathbf{R}_{j+1} A_{2}^{(j+2)}=0, \quad j \geq 1
$$

and the matrix sequence $\left\{\mathbf{G}_{j}, j \geq 2\right\}$ is the minimal nonnegative solution to the system of nonlinear matrix equations

$$
A_{0}^{(j)} \mathbf{G}_{j+1} \mathbf{G}_{j}+A_{1}^{(j)} \mathbf{G}_{j}+A_{2}^{(j)}=0, \quad j \geq 2 .
$$

Once the matrix sequence $\left\{\mathbf{R}_{j}, j \geq 1\right\}$ or $\left\{\mathbf{G}_{j}, j \geq 2\right\}$ is given, then for $j \geq 1$

$$
\begin{aligned}
\mathbf{U}_{j} & =A_{1}^{(j)}+A_{0}^{(j)}\left(-\mathbf{U}_{j+1}^{-1}\right) A_{2}^{(j+1)} \\
& =A_{1}^{(j)}+\mathbf{R}_{j} A_{2}^{(j+1)} \\
& =A_{1}^{(j)}+A_{0}^{(j)} \mathbf{G}_{j+1} .
\end{aligned}
$$

Based on this, we can provide the $U L$-type RG-factorization of the matrix $T$ of infinite size as follows:

$$
T=\left(I-\mathbf{R}_{U}\right) \mathbf{U}_{D}\left(I-\mathbf{G}_{L}\right)
$$

where

$$
I-\mathbf{R}_{U}=\left(\begin{array}{ccccc}
I & -\mathbf{R}_{1} & & & \\
& I & -\mathbf{R}_{2} & & \\
& & I & -\mathbf{R}_{3} & \\
& & & I & \ddots \\
& & & & \ddots
\end{array}\right)
$$




$$
\begin{gathered}
\mathbf{U}_{D}=\operatorname{diag}\left(\mathbf{U}_{1}, \mathbf{U}_{2}, \mathbf{U}_{3}, \ldots\right), \\
I-\mathbf{G}_{L}=\left(\begin{array}{ccccc}
I & & & & \\
-\mathbf{G}_{2} & I & & & \\
& -\mathbf{G}_{3} & I & & \\
& & -\mathbf{G}_{4} & I & \\
& & & \ddots & \ddots
\end{array}\right) .
\end{gathered}
$$

By using the $\mathbf{R}$-measure $\left\{\mathbf{R}_{j}: j \geq 1\right\}$ and the $\mathbf{G}$-measure $\left\{\mathbf{G}_{l}: l \geq 2\right\}$, we write

$$
\begin{gathered}
X_{j}^{(j)}=I, \\
X_{j+l}^{(j)}=\mathbf{R}_{j} \mathbf{R}_{j+1} \cdots \mathbf{R}_{j+l-1}, j \geq 1, l \geq 1, \\
Y_{j}^{(j)}=I, \\
Y_{j-l}^{(j)}=\mathbf{G}_{j} \mathbf{G}_{j-1} \cdots \mathbf{G}_{j-l+1}, j>l \geq 1 .
\end{gathered}
$$

The following lemma provides the inverses of the three matrices $I-\mathbf{R}_{U}, \mathbf{U}_{D}$ and $I-\mathbf{G}_{L}$, while their proofs are easy by means of $\left(I-\mathbf{R}_{U}\right)\left(I-\mathbf{R}_{U}\right)^{-1}=I, \mathbf{U}_{D} \mathbf{U}_{D}^{-1}=I$ and $\left(I-\mathbf{G}_{L}\right)\left(I-\mathbf{G}_{L}\right)^{-1}=I$.

Lemma 1. The matrices $I-\mathbf{R}_{U}, \mathbf{U}_{D}$ and $I-\mathbf{G}_{L}$ are invertible, and

$$
\begin{aligned}
& \left(I-\mathbf{R}_{U}\right)^{-1}=\left(\begin{array}{ccccc}
X_{1}^{(1)} & X_{2}^{(1)} & X_{3}^{(1)} & X_{4}^{(1)} & \ldots \\
& X_{2}^{(2)} & X_{3}^{(2)} & X_{4}^{(2)} & \ldots \\
& & X_{3}^{(3)} & X_{4}^{(3)} & \ldots \\
& & & X_{4}^{(4)} & \ldots \\
& & & & \ddots
\end{array}\right), \\
& \mathbf{U}_{D}^{-1}=\operatorname{diag}\left(\mathbf{U}_{1}^{-1}, \mathbf{U}_{2}^{-1}, \mathbf{U}_{3}^{-1}, \mathbf{U}_{4}^{-1}, \ldots\right), \\
& \left(I-\mathbf{G}_{L}\right)^{-1}=\left(\begin{array}{cccccc}
Y_{1}^{(1)} & & & & \\
Y_{1}^{(2)} & Y_{2}^{(2)} & & & \\
Y_{1}^{(3)} & Y_{2}^{(3)} & Y_{3}^{(3)} & & \\
Y_{1}^{(4)} & Y_{2}^{(4)} & Y_{3}^{(4)} & Y_{4}^{(4)} & \\
\vdots & \vdots & \vdots & \vdots & \ddots
\end{array}\right) .
\end{aligned}
$$


By using the RG-factorization given by (24), we obtain

$$
(-T)_{\min }^{-1}=-\left(I-\mathbf{G}_{L}\right)^{-1} \mathbf{U}_{D}^{-1}\left(I-\mathbf{R}_{U}\right)^{-1}
$$

Let

$$
(-T)_{\min }^{-1}=-\left(\begin{array}{ccccc}
t_{1}^{(1)} & t_{2}^{(1)} & t_{3}^{(1)} & t_{4}^{(1)} & \ldots \\
t_{1}^{(2)} & t_{2}^{(2)} & t_{3}^{(2)} & t_{4}^{(2)} & \ldots \\
t_{1}^{(3)} & t_{2}^{(3)} & t_{3}^{(3)} & t_{4}^{(3)} & \ldots \\
t_{1}^{(4)} & t_{2}^{(4)} & t_{3}^{(4)} & t_{4}^{(4)} & \ldots \\
\vdots & \vdots & \vdots & \vdots & \ddots
\end{array}\right),
$$

Then it follows from Lemma 1 that

$$
t_{n}^{(m)}= \begin{cases}\sum_{i=1}^{n} Y_{i}^{(m)} \mathbf{U}_{i}^{-1} X_{n}^{(i)}, & n<m \\ \sum_{i=1}^{n} Y_{i}^{(m)} \mathbf{U}_{i}^{-1} X_{n}^{(i)}, & n=m \\ \sum_{i=1}^{m} Y_{i}^{(m)} \mathbf{U}_{i}^{-1} X_{n}^{(i)}, & n>m .\end{cases}
$$

Thus it follows from (19) that

$$
\begin{aligned}
E\left[W^{(2)}\right] & =\vec{\alpha}(-T)_{\min }^{-1} \mathbf{e}=-\vec{\alpha}\left(I-\mathbf{G}_{L}\right)^{-1} \mathbf{U}_{D}^{-1}\left(I-\mathbf{R}_{U}\right)^{-1} \mathbf{e} \\
& =-\sum_{i=1}^{\infty} \sum_{j=1}^{\infty} \pi_{i} t_{j}^{(i)} \mathbf{e}
\end{aligned}
$$

\subsection{Sojourn Time of A-customers of Type 1}

When the double-ended queue is stable, we denote by $W^{(1)}$ the sojourn time of any arriving A-customer of Type 1.

Here, the state space of the Markov process are arranged in the following order:

$$
\left\{\mathbb{S}_{0}, \mathbb{S}_{1}, \mathbb{S}_{2}, \ldots\right\}, \mathbb{S}_{i}=\{(i, 0),(i, 1),(i, 2),(i, 3), \ldots\}
$$

Based on Figure 2, the infinitesimal generator of the QBD process $\left\{\left(N_{1}(t), N_{2}(t)\right), t \geq 0\right\}$ is given by

$$
Q=\left(\begin{array}{cccccc}
B_{1}^{(0)} & B_{0}^{(0)} & & & & \\
B_{2}^{(1)} & B_{1}^{(1)} & B_{0}^{(1)} & & & \\
& B_{2}^{(2)} & B_{1}^{(2)} & B_{0}^{(2)} & & \\
& & B_{2}^{(3)} & B_{1}^{(3)} & B_{0}^{(3)} & \\
& & & \ddots & \ddots & \ddots
\end{array}\right),
$$


where

$$
\begin{aligned}
& B_{0}^{(i)}=\left(\begin{array}{cccc}
\lambda_{1} & & & \\
& \lambda_{1} & & \\
& & \lambda_{1} & \\
& & & \ddots
\end{array}\right), i \geq 0
\end{aligned}
$$

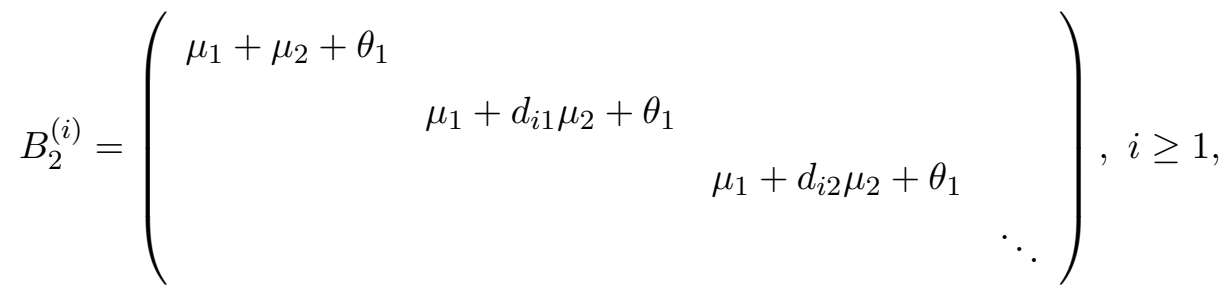

$$
\begin{aligned}
& B_{1}^{(0)}=\left(\begin{array}{ccccc}
B_{1,1} & \lambda_{2} & & \\
\mu_{2}+\theta_{2} & B_{1,2} & \lambda_{2} & & \\
& \mu_{2}+2 \theta_{2} & B_{1,3} & \lambda_{2} & \\
& & \ddots & \ddots & \ddots
\end{array}\right) \\
& B_{1, i}=-\left(\lambda_{1}+\lambda_{2}+\left(1-d_{0(i-1)}\right) \mu_{2}+(i-1) \theta_{2}\right), i \geq 1, d_{0 i}=\left\{\begin{array}{c}
1, i=0 \\
0, i \geq 1
\end{array},\right. \\
& B_{1}^{(i)}=\left(\begin{array}{ccccc}
B_{1,1} & \lambda_{2} & & \\
\left(1-d_{i 1}\right) \mu_{2}+\theta_{2} & B_{1,2} & \lambda_{2} & \\
& \left(1-d_{i 2}\right) \mu_{2}+2 \theta_{2} & B_{1,3} & \lambda_{2} & \\
& & \ddots & \ddots & \ddots
\end{array}\right), i \geq 1, \\
& B_{1, k}=-\left(\lambda_{1}+\lambda_{2}+\mu_{1}+\mu_{2}+i \theta_{1}+(k-1) \theta_{2}\right), k \geq 1 .
\end{aligned}
$$

Since the level-dependent QBD process is stable, we have

$$
h_{i, j}=\lim _{t \rightarrow+\infty} p_{i, j}(t) .
$$

For $k \geq 0$, we write

$$
h_{k}=\left(h_{k, 0}, h_{k, 1}, h_{k, 2}, \ldots\right) \text {, }
$$

and

$$
h=\left(h_{0}, h_{1}, h_{2}, \ldots\right) .
$$

Then we apply the RG-factorization given in $\mathrm{Li}[27]$ to calculate the stationary probability vector where the detailed analysis process is similar to section 4 . 
For a new arrival, each arriving A-customer of Type 1' sojourn time can be regarded as the first passage time that the QBD process $\left\{\left(N_{1}(t), N_{2}(t)\right), t \geq 0\right\}$ with absorbing states $\{(0,0),(0,1),(0,2), \ldots\}$ and on a state sub-space

$$
\{\{1,2,3, \ldots\} \times\{0,1,2, \ldots\}\}
$$

begins from state $(i, j)$ with probability $h_{i, j}$ for $i \geq 1, j \geq 0$ until it finally enters the absorption state $\{(0,0),(0,1),(0,2), \ldots\}$. Note that the new QBD process with the absorption state $\{(0,0),(0,1),(0,2), \ldots\}$ is depicted in Figure 4 , thus the infinitesimal generator of the new QBD process with the absorption state $\{(0,0),(0,1),(0,2), \ldots\}$ is given by

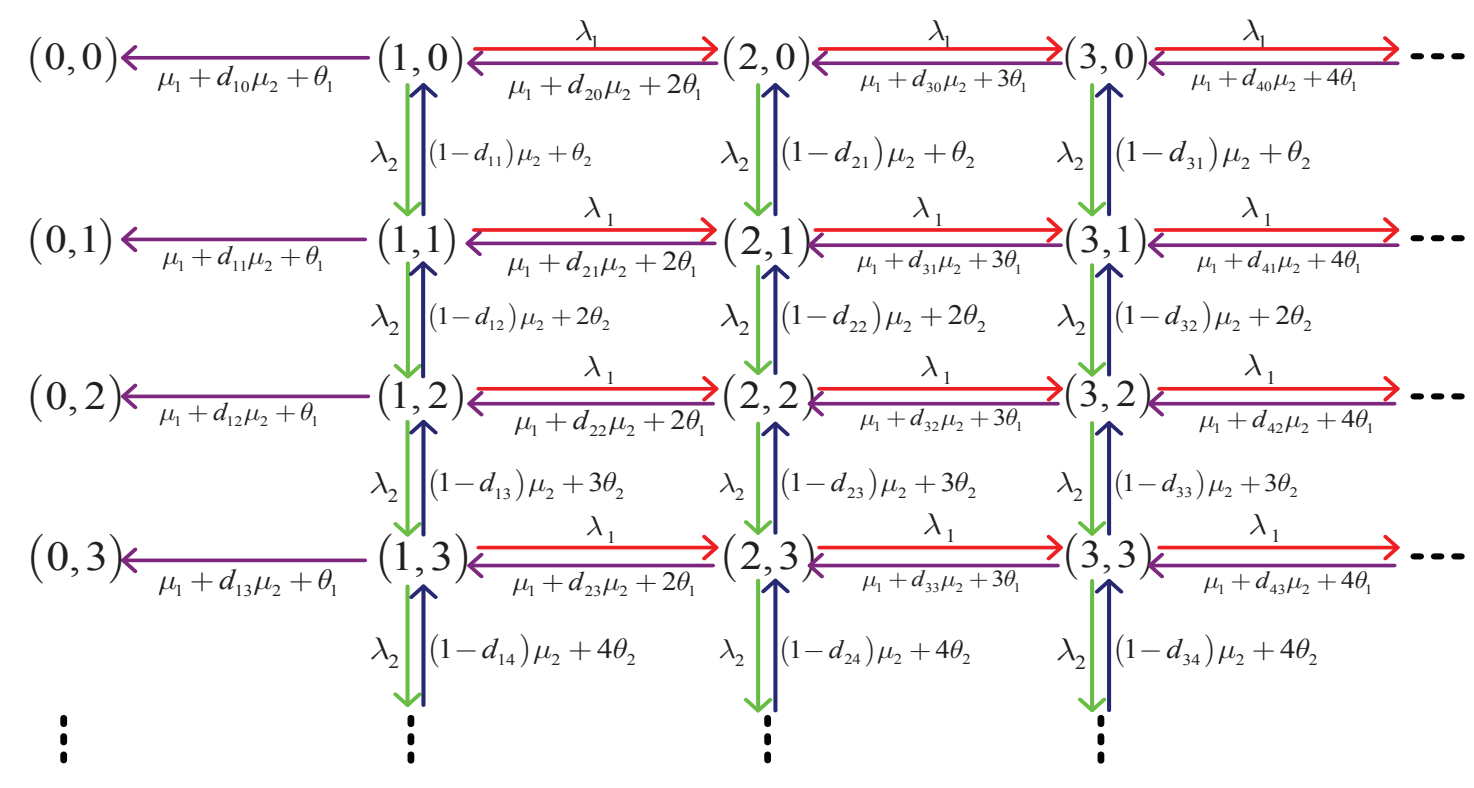

Figure 4: The state transition relations of the QBD process

$$
\Xi=\left(\begin{array}{cccccc}
0 & \mathbf{0} & \mathbf{0} & \mathbf{0} & \mathbf{0} & \boldsymbol{\cdots} \\
B_{2}^{(1)} \mathbf{e} & B_{1}^{(1)} & B_{0}^{(1)} & & & \\
& B_{2}^{(2)} & B_{1}^{(2)} & B_{0}^{(2)} & & \\
& & B_{2}^{(3)} & B_{1}^{(3)} & B_{0}^{(3)} & \\
& & & \ddots & \ddots & \ddots
\end{array}\right)
$$


where

$$
\begin{aligned}
& B_{0}^{(i)}=\left(\begin{array}{cccc}
\lambda_{1} & & & \\
& \lambda_{1} & & \\
& & \lambda_{1} & \\
& & & \ddots
\end{array}\right), i \geq 1
\end{aligned}
$$

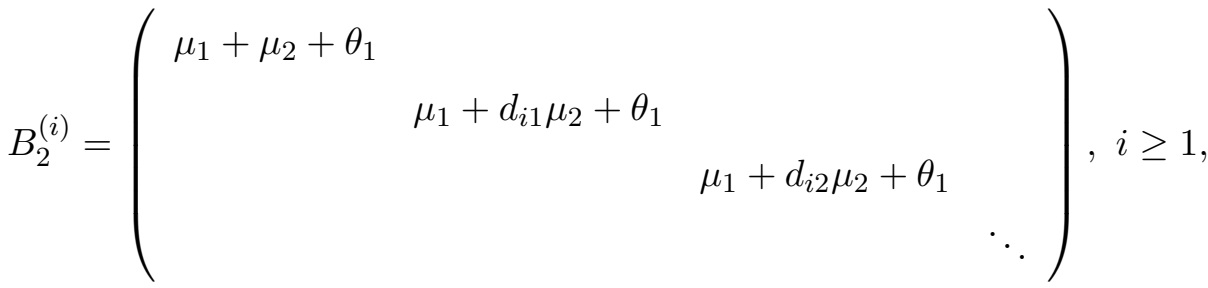

$$
\begin{aligned}
& B_{1}^{(i)}=\left(\begin{array}{ccccc}
B_{1,1} & \lambda_{2} & & \\
\left(1-d_{i 1}\right) \mu_{2}+\theta_{2} & B_{1,2} & \lambda_{2} & \\
& \left(1-d_{i 2}\right) \mu_{2}+2 \theta_{2} & B_{1,3} & \lambda_{2} & \\
& & \ddots & \ddots & \ddots
\end{array}\right), i \geq 1, \\
& B_{1, k}=-\left(\lambda_{1}+\lambda_{2}+\mu_{1}+\mu_{2}+i \theta_{1}+(k-1) \theta_{2}\right), k \geq 1,
\end{aligned}
$$

Let

$$
\mathbb{T}=\left(\begin{array}{cccccc}
B_{1}^{(1)} & B_{0}^{(1)} & & & & \\
B_{2}^{(2)} & B_{1}^{(2)} & B_{0}^{(2)} & & & \\
& B_{2}^{(3)} & B_{1}^{(3)} & B_{0}^{(3)} & & \\
& & B_{2}^{(4)} & B_{1}^{(4)} & B_{0}^{(4)} & \\
& & & \ddots & \ddots & \ddots
\end{array}\right), \quad \mathbb{T}^{0}=\left(\begin{array}{c}
B_{2}^{(1)} \mathbf{e} \\
0 \\
0 \\
0 \\
\vdots
\end{array}\right) .
$$

Then (32) can be rewritten as

$$
\Xi=\left(\begin{array}{cc}
0 & 0 \\
\mathbb{T}^{0} & \mathbb{T}
\end{array}\right)
$$

Here, $\mathbb{T e}+\mathbb{T}^{0}=0$.

Let $\left(\gamma_{0}, \vec{\gamma}\right)$ denote the initial probability distribution of the QBD process $\Xi$ with the absorption state $\{(0,0),(0,1),(0,2), \ldots\}$, where $\vec{\gamma}=\left(h_{1}, h_{2}, \ldots\right)$ and $\gamma_{0}=1-\vec{\gamma} \mathbf{e}=h_{0}$.

The following theorem uses the phase-type distribution of infinite size to provide expression for the probability distribution of the sojourn time $W^{(1)}$. 
Theorem 4. If the initial probability distribution of the $Q B D$ process $\Xi$ with the absorption state $\{(0,0),(0,1),(0,2), \ldots\}$ is $\left(\gamma_{0}, \vec{\gamma}\right)$, then the probability distribution of the sojourn time $W^{(1)}$ is phase-type with an irreducible representation $(\vec{\gamma}, \mathbb{T})$, that is,

$$
F_{W^{(1)}}(t)=P\left(W^{(1)} \leq t\right)=1-\vec{\gamma} \exp (\mathbb{T} t) \mathbf{e}, \quad t \geq 0 .
$$

Proof. For $i=1,2,3, \ldots, j=0,1,2, \ldots$, we write

$$
q_{i, j}(t)=P\left\{N_{1}(t)=i, N_{2}(t)=j\right\}
$$

for $i=1,2,3, \ldots$, we write

$$
q_{i}(t)=\left(q_{i, 0}(t), q_{i, 1}(t), q_{i, 2}(t), \ldots\right)
$$

and

$$
q(t)=\left(q_{1}(t), q_{2}(t), q_{3}(t), \ldots\right) .
$$

For the probability vector $q(t)$, it follows from the Chapman-Kolmogorov forward equation that

$$
\frac{d}{d t} q(t)=q(t) \mathbb{T}
$$

with the initial condition

$$
q(0)=\vec{\gamma}
$$

It follows from (34) and (35) that

$$
q(t)=\vec{\gamma} e^{\mathbb{T} t}
$$

Thus, we obtain

$$
F_{W^{(1)}}(t)=P\left(W^{(1)} \leq t\right)=1-q(t) \mathbf{e}=1-\vec{\gamma} \exp (\mathbb{T} t) \mathbf{e}, t \geq 0 .
$$

This completes the proof.

In the remainder of this section, we compute the mean $E\left[W^{(1)}\right]$ of the sojourn time $W^{(1)}$ of each arriving A-customer of Type 1 by means of the Laplace-Stieltjes transform and the RG-factorization.

We denote by $f(s)$ the Laplace-Stieltjes transform of the distribution function $F_{W^{(1)}}(t)$ or the random variable $W^{(1)}$. Thus, we have 


$$
\begin{aligned}
f(s) & =\int_{0}^{\infty} e^{-s t} d F_{W^{(1)}}(t) \\
& =1-\vec{\gamma} \mathbf{e}+\vec{\gamma}(s I-\mathbb{T})_{\min }^{-1} \mathbb{T}^{0},
\end{aligned}
$$

where $I$ denotes an identity matrix of infinite size, and $(s I-\mathbb{T})_{\min }^{-1}$ is the minimal nonnegative inverse of the matrix $s I-\mathbb{T}$ of infinite size. See Li and Cao [28] and Li [27] for more details. It is easy to see that

$$
E\left[W^{(1)}\right]=-\frac{d}{d s} f(s)_{\mid s=0}=\vec{\gamma}\left[(s I-\mathbb{T})_{\min }^{-2}\right]_{\mid s=0} \mathbb{T}^{0}=\vec{\gamma}(-\mathbb{T})_{\min }^{-1} \mathbf{e}
$$

by using $\mathbb{T} \mathbf{e}+\mathbb{T}^{0}=0$ and $(-\mathbb{T})_{\min }^{-1}(-\mathbb{T}) \mathbf{e}=\mathbf{e}$.

In computation of the mean $E\left[W^{(1)}\right]$, it is easy to see that the key is how to deal with the minimal nonnegative inverse $(-\mathbb{T})_{\min }^{-1}$ of the matrix $-\mathbb{T}$ of infinite size. To this end, we develop an effective procedure to compute the minimal nonnegative inverse $(-\mathbb{T})_{\min }^{-1}$ by means of the RG-factorization.

For the QBD process $\mathbb{T}$, we define the $U L$-type $\mathbb{U}$-, $\mathbb{R}$ - and $\mathbb{G}$-measures as

$$
\begin{gathered}
\mathbb{U}_{k}=B_{1}^{(k)}+B_{0}^{(k)}\left(-\mathbb{U}_{k+1}^{-1}\right) B_{2}^{(k+1)}, \quad k \geq 1, \\
\mathbb{R}_{k}=B_{0}^{(k)}\left(-\mathbb{U}_{k+1}^{-1}\right), \quad k \geq 1,
\end{gathered}
$$

and

$$
\mathbb{G}_{k}=\left(-\mathbb{U}_{k}^{-1}\right) B_{2}^{(k)}, \quad k \geq 2 .
$$

On the other hand, note that the matrix sequence $\left\{\mathbb{R}_{k}, k \geq 1\right\}$ is the minimal nonnegative solution to the system of nonlinear matrix equations

$$
B_{0}^{(k)}+\mathbb{R}_{k} B_{1}^{(k+1)}+\mathbb{R}_{k} \mathbb{R}_{k+1} B_{2}^{(k+2)}=0, \quad k \geq 1,
$$

and the matrix sequence $\left\{\mathbb{G}_{k}, k \geq 2\right\}$ is the minimal nonnegative solution to the system of nonlinear matrix equations

$$
B_{0}^{(k)} \mathbb{G}_{k+1} \mathbb{G}_{k}+B_{1}^{(k)} \mathbb{G}_{k}+B_{2}^{(k)}=0, \quad k \geq 2 .
$$

Once the matrix sequence $\left\{\mathbb{R}_{k}, k \geq 1\right\}$ or $\left\{\mathbb{G}_{k}, k \geq 2\right\}$ is given, then for $k \geq 1$

$$
\begin{aligned}
\mathbb{U}_{k} & =B_{1}^{(k)}+B_{0}^{(k)}\left(-\mathbb{U}_{k+1}^{-1}\right) B_{2}^{(k+1)} \\
& =B_{1}^{(k)}+\mathbb{R}_{k} B_{2}^{(k+1)} \\
& =B_{1}^{(k)}+B_{0}^{(k)} \mathbb{G}_{k+1} .
\end{aligned}
$$


Based on this, we can provide the $U L$-type $R G$-factorization of the matrix $\mathbb{T}$ of infinite size as follows:

$$
\mathbb{T}=\left(I-\mathbb{R}_{U}\right) \mathbb{U}_{D}\left(I-\mathbb{G}_{L}\right),
$$

where

$$
\begin{aligned}
& I-\mathbb{R}_{U}=\left(\begin{array}{ccccc}
I & -\mathbb{R}_{1} & & & \\
& I & -\mathbb{R}_{2} & & \\
& & I & -\mathbb{R}_{3} & \\
& & & I & \ddots \\
& & & & \ddots
\end{array}\right) \\
& \mathbb{U}_{D}=\operatorname{diag}\left(\mathbb{U}_{1}, \mathbb{U}_{2}, \mathbb{U}_{3}, \ldots\right) \text {, } \\
& I-\mathbb{G}_{L}=\left(\begin{array}{ccccc}
I & & & & \\
-\mathbb{G}_{2} & I & & & \\
& -\mathbb{G}_{3} & I & & \\
& & -\mathbb{G}_{4} & I & \\
& & & \ddots & \ddots
\end{array}\right) .
\end{aligned}
$$

By using the $\mathbb{R}$-measure $\left\{\mathbb{R}_{k}: k \geq 1\right\}$ and the $\mathbb{G}$-measure $\left\{\mathbb{G}_{l}: l \geq 2\right\}$, we write

$$
\begin{gathered}
X_{k}^{(k)}=I, \\
X_{k+l}^{(k)}=\mathbb{R}_{k} \mathbb{R}_{k+1} \cdots \mathbb{R}_{k+l-1}, k \geq 1, l \geq 1, \\
Y_{k}^{(k)}=I, \\
Y_{k-l}^{(k)}=\mathbb{G}_{k} \mathbb{G}_{k-1} \cdots \mathbb{G}_{k-l+1}, k>l \geq 1 .
\end{gathered}
$$

The following lemma provides the inverses of the three matrices $I-\mathbb{R}_{U}, \mathbb{U}_{D}$ and $I-\mathbb{G}_{L}$, while their proofs are easy by means of $\left(I-\mathbb{R}_{U}\right)\left(I-\mathbb{R}_{U}\right)^{-1}=I, \mathbb{U}_{D} \mathbb{U}_{D}^{-1}=I$ and $\left(I-\mathbb{G}_{L}\right)\left(I-\mathbb{G}_{L}\right)^{-1}=I$.

Lemma 2. The matrices $I-\mathbb{R}_{U}, \mathbb{U}_{D}$ and $I-\mathbb{G}_{L}$ are invertible, and

$$
\left(I-\mathbb{R}_{U}\right)^{-1}=\left(\begin{array}{ccccc}
X_{1}^{(1)} & X_{2}^{(1)} & X_{3}^{(1)} & X_{4}^{(1)} & \ldots \\
& X_{2}^{(2)} & X_{3}^{(2)} & X_{4}^{(2)} & \ldots \\
& & X_{3}^{(3)} & X_{4}^{(3)} & \ldots \\
& & & X_{4}^{(4)} & \ldots \\
& & & & \ddots
\end{array}\right)
$$




$$
\begin{gathered}
\mathbb{U}_{D}^{-1}=\operatorname{diag}\left(\mathbb{U}_{1}^{-1}, \mathbb{U}_{2}^{-1}, \mathbb{U}_{3}^{-1}, \mathbb{U}_{4}^{-1}, \ldots\right), \\
\left(I-\mathbb{G}_{L}\right)^{-1}=\left(\begin{array}{ccccc}
Y_{1}^{(1)} & & & & \\
Y_{1}^{(2)} & Y_{2}^{(2)} & & & \\
Y_{1}^{(3)} & Y_{2}^{(3)} & Y_{3}^{(3)} & & \\
Y_{1}^{(4)} & Y_{2}^{(4)} & Y_{3}^{(4)} & Y_{4}^{(4)} & \\
\vdots & \vdots & \vdots & \vdots & \ddots
\end{array}\right) .
\end{gathered}
$$

By using the RG-factorization given by (44), we obtain

$$
(-\mathbb{T})_{\min }^{-1}=-\left(I-\mathbb{G}_{L}\right)^{-1} \mathbb{U}_{D}^{-1}\left(I-\mathbb{R}_{U}\right)^{-1}
$$

Let

$$
(-\mathbb{T})_{\min }^{-1}=-\left(\begin{array}{ccccc}
t_{1}^{(1)} & t_{2}^{(1)} & t_{3}^{(1)} & t_{4}^{(1)} & \ldots \\
t_{1}^{(2)} & t_{2}^{(2)} & t_{3}^{(2)} & t_{4}^{(2)} & \ldots \\
t_{1}^{(3)} & t_{2}^{(3)} & t_{3}^{(3)} & t_{4}^{(3)} & \ldots \\
t_{1}^{(4)} & t_{2}^{(4)} & t_{3}^{(4)} & t_{4}^{(4)} & \ldots \\
\vdots & \vdots & \vdots & \vdots & \ddots
\end{array}\right)
$$

Then it follows from Lemma 2 that

$$
t_{n}^{(m)}= \begin{cases}\sum_{i=1}^{n} Y_{i}^{(m)} \mathbb{U}_{i}^{-1} X_{n}^{(i)}, & n<m \\ \sum_{i=1}^{n} Y_{i}^{(m)} \mathbb{U}_{i}^{-1} X_{n}^{(i)}, & n=m \\ \sum_{i=1}^{m} Y_{i}^{(m)} \mathbb{U}_{i}^{-1} X_{n}^{(i)}, & n>m\end{cases}
$$

Thus it follows from (39) that

$$
\begin{aligned}
E\left[W^{(1)}\right] & =\vec{\gamma}(-\mathbb{T})_{\min }^{-1} \mathbf{e}=-\vec{\gamma}\left(I-\mathbb{G}_{L}\right)^{-1} \mathbb{U}_{D}^{-1}\left(I-\mathbb{R}_{U}\right)^{-1} \mathbf{e} \\
& =-\sum_{i=1}^{\infty} \sum_{j=1}^{\infty} h_{i} t_{j}^{(i)} \mathbf{e} .
\end{aligned}
$$

\section{$6 \quad$ Numerical Examples}

In this section, by applying the RG-factorizations and using efficient procedures given by Bright and Taylor $[7,8]$, we give some numerical examples to verify the correctness of our theoretical results, and analyze how the stationary queue lengths and the sojourn time of the double-ended queue are influenced by key system parameters $\theta_{1}, \theta_{2}$ (i.e., the impatient 
rates of A-customers of Type 1 and Type 2), respectively. At the same time, we also apply the coupling method to provide some interesting interpretations on the numerical analysis.

For the A-customers of Type 1 and Type 2, and the B-customers of Type 1 and Type 2 , we respectively take their arrival rates as follows:

$$
\lambda_{1}=10, \lambda_{2}=10, \mu_{1}=5, \mu_{2}=5 .
$$

And we assume that the waiting spaces for A-customers and B-customers are $M_{1}$ and $M_{2}$, and we respectively take $M_{1}$ and $M_{2}$ as follows:

$$
M_{1}=100, M_{2}=100
$$

We discuss that how $E\left[\mathcal{Q}^{(1)}\right], E\left[\mathcal{Q}^{(2)}\right], E\left[W^{(1)}\right]$ and $E\left[W^{(2)}\right]$ depend on the two impatient rates $\theta_{1}$ and $\theta_{2}$ when $\theta_{2}=2,4,5, \theta_{1} \in[2,5]$ and $\theta_{1}=3,4,5, \theta_{2} \in[2,4]$, respectively.

Figure 5 shows that the average stationary queue length $E\left[\mathcal{Q}^{(1)}\right]$ decreases as $\theta_{1}$ and $\theta_{2}$ increase. From Figure 6, we observe that the average stationary queue length $E\left[\mathcal{Q}^{(2)}\right]$ decreases as $\theta_{1}$ and $\theta_{2}$ increase.
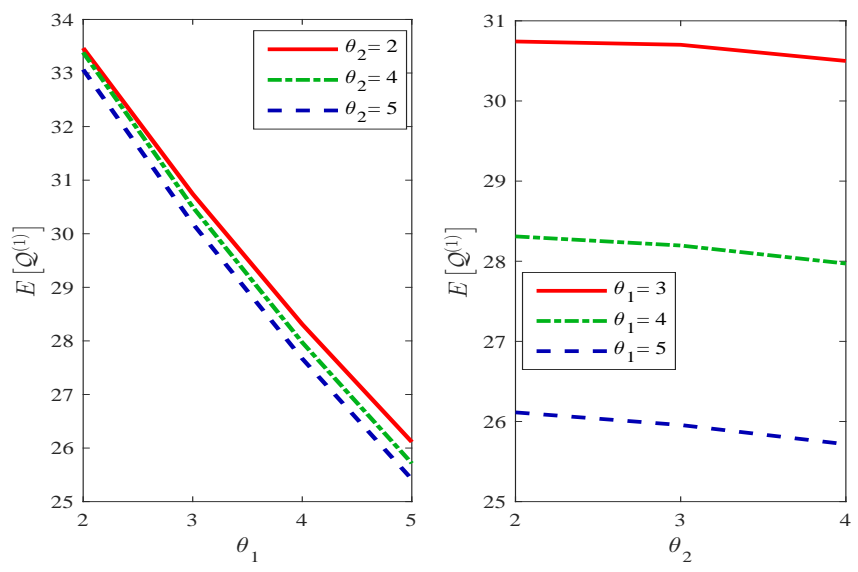

Figure 5: $E\left[\mathcal{Q}^{(1)}\right]$ vs $\theta_{1}$ and $\theta_{2}$

The four numerical results are intuitive. As $\theta_{1}$ increases, more and more A-customers of Type 1 are quickly leaving the system so that the chance that an A-customer of Type 2 can match a B-customer of type 2 will become bigger. Thus, $E\left[\mathcal{Q}^{(2)}\right]$ decreases as $\theta_{1}$ increases. On the other hand, as $\theta_{2}$ increases, more and more A-customers of Type 2 are 

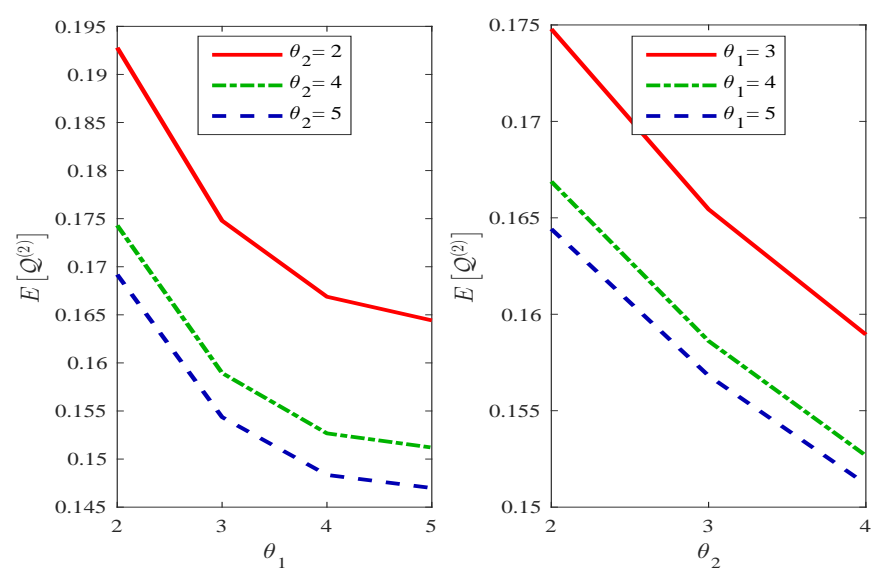

Figure 6: $E\left[\mathcal{Q}^{(2)}\right]$ vs $\theta_{1}$ and $\theta_{2}$

quickly leaving the system so that the chance that an A-customer of Type 1 can match a B-customer of type 2 will become bigger. Thus, $E\left[\mathcal{Q}^{(1)}\right]$ decreases as $\theta_{2}$ increases.
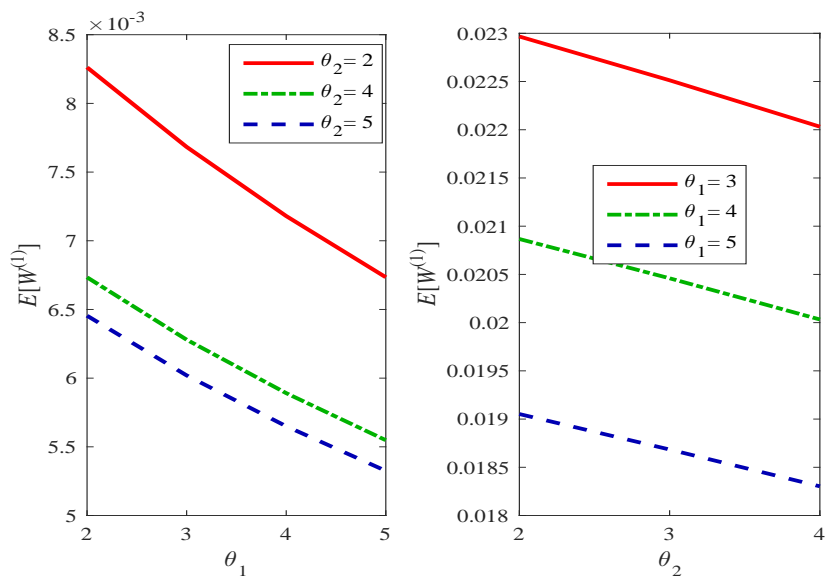

Figure 7: $E\left[W^{(1)}\right]$ vs $\theta_{1}$ and $\theta_{2}$

Figure 7 indicates that the sojourn time $E\left[W^{(1)}\right]$ decreases as $\theta_{1}$ and $\theta_{2}$ increase. Figure 8 shows that the sojourn time $E\left[W^{(2)}\right]$ decreases as $\theta_{1}$ and $\theta_{2}$ increase.

\section{Concluding Remarks}

In this paper, we describe a double-ended queue with flexible customers, four Poisson inputs, and customers' impatient behaviors, and show that such a queue can be expressed 

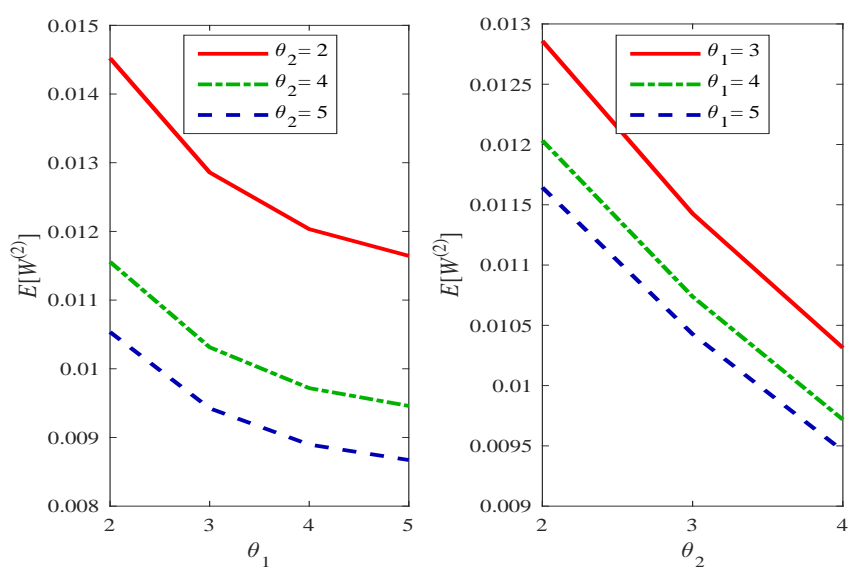

Figure 8: $E\left[W^{(2)}\right]$ vs $\theta_{1}$ and $\theta_{2}$

as a QBD process with infinitely many phases. Based on this finding, we provide a detailed analysis for the double-ended queue, including the system stability, the stationary probability vector, the sojourn time, and so forth. Finally, some numerical examples are given to verify the correctness of our theoretical results, and demonstrate how the performance measures of this queue are influenced by key system parameters. We believe that the methodology and results proposed in this paper can be applicable to deal with more general matching queues.

Along these lines, we will continue our future research on the following directions:

- Introduce fluid and diffusion limits to study the system of this paper.

- Develop stochastic optimization and control, Markov decision processes and stochastic game theory in the study of double-ended queues with flexible customers.

- Consider an extension of the system in which the B-customers can queue.

Author Contributions: Conceptualization, methodology, software, validation, formal analysis, investigation, resources, writing - original draft preparation, writing - review and editing, visualization, supervision, H.L.Liu. All authors have read and agreed to the published version of the manuscript.

Funding: This research received no external funding.

Acknowledgments: The authors would like to thank the anonymous reviewers for their constructive suggestions.

Conflicts of Interest: The authors declare no conflict of interest. 


\section{References}

[1] Adan I, Weiss G (2012) Exact fcfs matching rates for two infinite multitype sequences. Operations Research 60(2): 475-489.

[2] Axsäter S (2015) Inventory Control, volume 225 (Springer).

[3] Baik H, Sherali HD, Trani AA (2002) Time-dependent network assignment strategy for taxiway routing at airports. Transportation research record 1788(1):70-75.

[4] Benjaafar S, Hu M (2020) Operations management in the age of the sharing economy: What is old and what is new? Manufacturing and Service Operations Management 22(1):93-101.

[5] Bhat UN (1970) A controlled transportation queueing process. Management Science 16(7):446-452.

[6] Boxma OJ, David I, Perry D, Stadje W (2011) A new look at organ transplantation models and double matching queues. Probability in the Engineering and Informational Sciences 25(2):135-155.

[7] Bright L, Taylor PG (1995) Calculating the equilibrium distribution in level dependent quasi-birth-and-death processes. Stochastic Models 11(3):497-525.

[8] Bright L, Taylor PG (1997) Equilibrium distributions for level-dependent quasi-birthand-death processes. In: Matrix-Analytic Methods in Stochastic Models, Pages 359-375. Marcel Dekker.

[9] Browne JJ, Kelly JJ, Le Bourgeois (1970) Maximum inventories in baggage claim: a double ended queuing system. Transportation Science 4(1):64-78.

[10] Burak B, Chen H (2015) Stabilizing policies for probabilistic matching systems[J]. Queueing Systems 80(1-2).

[11] Büke B, Chen H (2017) Fluid and diffusion approximations of probabilistic matching systems. Queueing Systems 86:1-33.

[12] Bušić A, Gupta V, Mairesse J (2013) Stability of the bipartite matching model. Advances in Applied Probability 45(2):351-378. 
[13] Caldentey R, Kaplan E, Weiss G (2009) FCFS infinite bipartite matching of servers and customers. Advances in Applied Probability 41(3):695-730.

[14] Castro F, Nazerzadeh H, Yan C (2020) Matching queues with reneging: a product form solution. Queueing Systems 96(3-4): 359-385.

[15] Chen N, Immorlica N, Karlin AR, Mahdian M, Rudra A (2009) Approximating matches made in heaven. lecture notes in computer science 5555:266-278.

[16] Cheng M (2016) Sharing economy: A review and agenda for future research. International Journal of Hospitality Management 57(1):60-70.

[17] Conolly BW, Parthasarathy PR, Selvaraju N (2002) Double-ended queues with impatience. Computers \& Operations Research 29(14):2053-2072.

[18] Elalouf A, Perlman Y, Yechiali U (2018) A double-ended queueing model for dynamic allocation of live organs based on a best-fit criterion. Applied Mathematical Modelling 60:179-191.

[19] Epstein, Robert (2009) The Truth about Online Dating. entific American Mind 20(3):54-61.

[20] Giveen SM (1961) A taxicab problem considered as a double-ended queue. Operations Research, Vol. 9, B44.

[21] Giveen SM (1963) A taxicab problem with time-dependent arrival rates. SIAM Review $5(2): 119-127$.

[22] Hopp WJ, Simon JT (1989) Bounds and heuristics for assembly-like queues. Queueing systems 4(2):137-155.

[23] Johnson M P (2003) Single-Period Location Models for Subsidized Housing: TenantBased Subsidies. Annals of Operations Research 123(Oct):105-124.

[24] Kashyap BRK (1965) A double-ended queueing system with limited waiting space. Proc. Nat. Inst. Sci. India 31(6): 559-570.

[25] Kashyap BRK (1966) The double-ended queue with bulk service and limited waiting space. Operations Research 14(5):822-834. 
[26] Kashyap BRK (1967) Further results for the double ended queue. Metrika 11(1):168186.

[27] Li QL (2010) Constructive Computation in Stochastic Models with Applications: The $R G$-Factorizations. Springer.

[28] Li QL, Cao J (2004) Two types of RG-factorizations of quasi-birth-and-death processes and their applications to stochastic integral functionals. Stochastic Models 20(3):299-340.

[29] Liu H L, Li Q L, Chang Y X, Zhang C (2020) Block-Structured Double-Ended Queues and Bilateral QBD Processes. arXiv preprint arXiv:2001.00946.

[30] Liu H L, Li Q L, Zhang C (2020) Matched Queues with Matching Batch Pair (m, n). arXiv preprint arXiv:2009.02742.

[31] Liu X, Gong Q, Kulkarni VG (2015) Diffusion models for double-ended queues with renewal arrival processes. Stochastic Systems.

[32] Mairesse J, Moyal P (2016) Stability of the stochastic matching model. Journal of Applied Probability 53(4):1064-1077.

[33] Neuts MF (1981) Matrix-Geometric Solutions in Stochastic Models: An Algorithmic Approach. Johns Hopkins University Press.

[34] Pandey MK, Gangeshwer DK (2018) Applications of the diffusion approximation to hospital sector using G1/GM/1 double ended queue model. Journal of Computer and Mathematical Sciences 9(4):302-308.

[35] Parthasarathy PR, Selvaraju N, Manimaran G (1999) A paired queueing system arising in multimedia synchronization. Mathematical and Computer Modelling 30(1112):133-140.

[36] Perlman Y, Elalouf A, Yechiali U (2018) Dynamic allocation of stochastically-arriving flexible resources to random streams of objects with application to kidney crosstransplantation. European Journal of Operational Research 265(1): 169-177.

[37] Pizzato L, Rej T, Akehurst J, Koprinska I, Yacef K, Kay J (2013) Recommending people to people: the nature of reciprocal recommenders with a case study in online dating. User Modeling and User-Adapted Interaction 23(5):447-488. 
[38] Porteus EL (1990) Stochastic inventory theory. Handbooks in Operations Research and Management Science 2:605-652.

[39] Ramachandran S, Delen D (2005). Performance analysis of a kitting process in stochastic assembly systems. Computers $\mathcal{E}$ Operations Research 32(3):449-463.

[40] Sasieni MW (1961) Double queues and impatient customers with an application to inventory theory. Operations Research 9(6):771-781.

[41] Shi Y, Lian Z (2016) Optimization and strategic behavior in a passenger-taxi service system. European Journal of Operational Research 249(3):1024-1032.

[42] Som P, Wilhelm WE, Disney RL (1994) Kitting process in a stochastic assembly system. Queueing Systems 17(3-4):471-490.

[43] Stanford DA, Lee JM, Chandok N, McAlister V (2014) A queuing model to address waiting time inconsistency in solid-organ transplantation. Operations Research for Health Care 3(1):40-45.

[44] Steinmetz R (1990) Synchronization properties in multimedia systems. IEEE Journal on Selected Areas in Communications 8(3):401-412.

[45] Sutherland W, Jarrahi MH (2018) The sharing economy and digital platforms: A review and research agenda. International Journal of Information Management 43:328341.

[46] Talreja R, Whitt W (2008) Fluid models for overloaded multiclass many-server queueing systems with first-come, first-served routing. Management Science 54(8):1513-1527.

[47] Ward A R, Gurvich I (2015) On the dynamic control of matching queues. Stochastic Systems 4(2).

[48] Zenios SA (1999) Modeling the transplant waiting list: A queueing model with reneging. Queueing Systems 31(3-4):239-251.

[49] Zhang W, Honnappa H, Ukkusuri SV (2019) Modeling urban taxi services with ehailings: A queueing network approach. Transportation Research Part C: Emerging Technologies 113:332-349. 